\title{
Tumor clonality and resistance mechanisms in EGFR mutation-positive non-small-cell lung cancer: implications for therapeutic sequencing
}

\author{
Shinji Kohsaka*,1, Mark Petronczki², Flavio Solca ${ }^{2}$ \& Makoto Maemondo $^{3}$ \\ ${ }^{1}$ Division of Cellular Signaling, National Cancer Center Research Institute, Tokyo, Japan \\ ${ }^{2}$ Boehringer Ingelheim RCV GmbH \& Co KG, Vienna, Austria \\ ${ }^{3}$ Department of Internal Medicine, Iwate Medical University, Morioka, Japan \\ *Author for correspondence: Tel.: +81 33547 5201; Fax: +81 35565 0727; skohsaka@ncc.go.jp
}

While the development of EGFR-targeted tyrosine kinase inhibitors (TKIs) has revolutionized treatment of EGFR mutation-positive non-small-cell lung cancer, acquired resistance to therapy is inevitable, reflecting tumor evolution. Recent studies show that EGFR mutation-positive non-small-cell lung cancer is highly heterogeneous at the cellular level, facilitating clonal expansion of resistant tumors via multiple molecular mechanisms. Here, we review the mechanistic differences between first-, second- and third-generation EGFR-targeted TKIs and speculate how these features could explain differences in clinical activity between these agents from a clonal evolution perspective. We hypothesize that the molecular dissection of tumor resistance mechanisms will facilitate optimal sequential use of EGFR TKIs in individual patients, thus maximizing the duration of chemotherapy-free treatment and survival benefit.

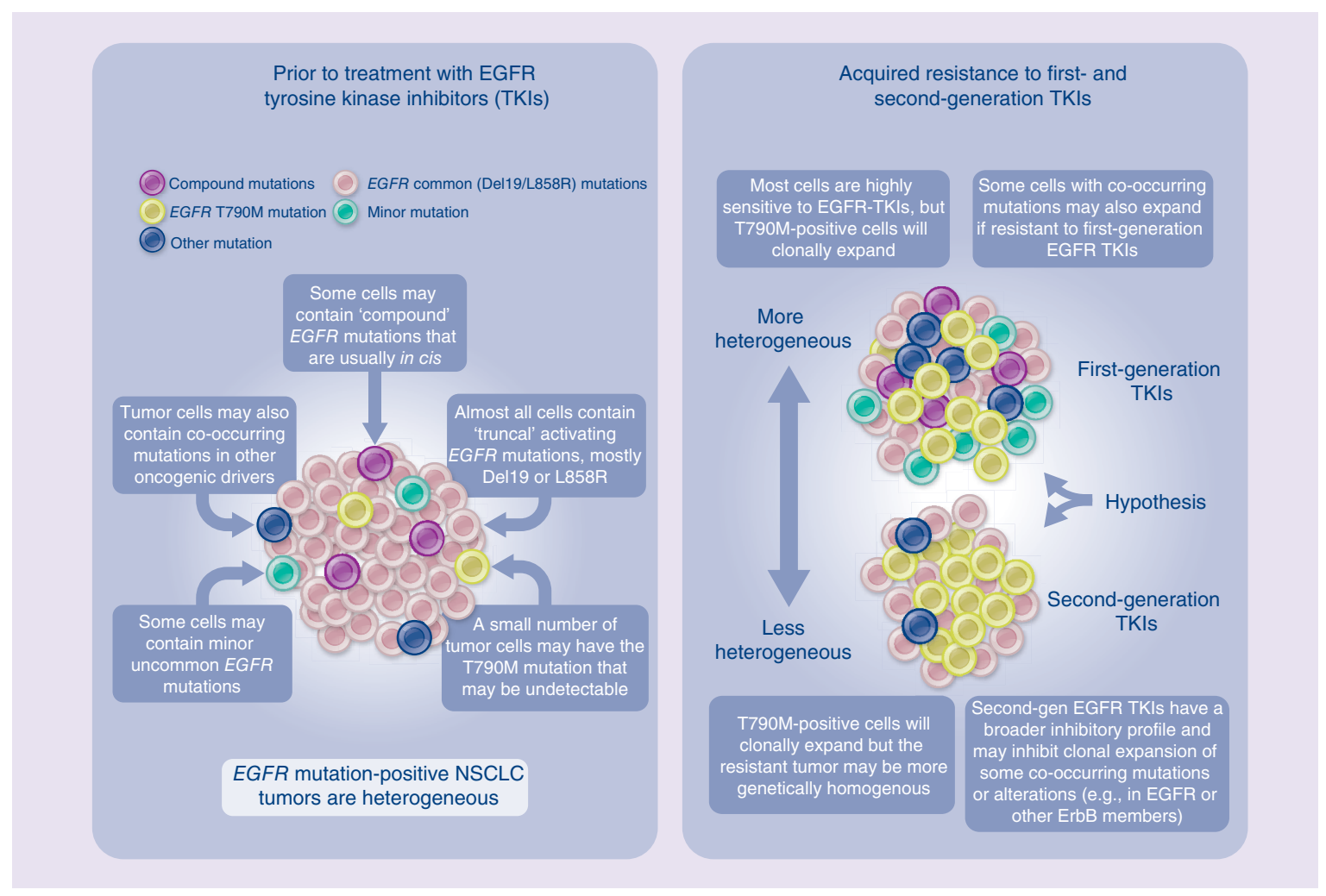

Future Medicine 
First draft submitted: 25 September 2018; Accepted for publication: 19 October 2018; Published online: 8 November 2018

\section{Keywords: acquired resistance $\bullet$ afatinib $\bullet$ clonal evolution $\bullet$ EGFR $\bullet$ erlotinib $\bullet$ gefitinib $\bullet$ NSCLC $\bullet$ osimertinib}

Patients with EGFR mutation-positive non-small-cell lung cancer (NSCLC) are generally highly responsive to treatment with EGFR-targeted tyrosine kinase inhibitors (TKIs). Several TKIs are approved for the treatment of EGFR mutation-positive NSCLC, and are categorized as first-, second- and third-generation agents based on profound differences in pharmacological characteristics, mechanism-of-action and the order in which they were developed. First-generation TKIs, such as gefitinib and erlotinib, non-covalently and reversibly bind to EGFR (both wild-type and mutated forms). They interrupt EGFR signaling by competing with ATP at the intracellular kinase domain of the receptor, thus inhibiting dimer autophosphorylation and attenuating intracellular signaling [1,2]. Several seminal Phase III trials have demonstrated that first-line gefitinib and erlotinib prolong progression-free survival (PFS), but not overall survival (OS), compared with platinum-doublet chemotherapy in patients with $E G F R$ mutation-positive NSCLC (median PFS of 9.2-13.1 months vs 4.6-6.3 months, respectively) [3-11]. Recent studies have demonstrated that the activity of first-generation TKIs is accentuated by combining them with anti-angiogenic agents such as bevacizumab [12].

The second-generation EGFR-targeted TKI, afatinib, works differently to erlotinib and gefitinib. It covalently and irreversibly binds not only to EGFR but also to other members of the ErbB family of receptors, HER2 and HER4, thus blocking transphosphorylation of tyrosine residues at the C-terminus of all three receptors [13,14]. It also blocks the transphosphorylation of the kinase inactive HER3 protein via its partner in HER3-containing heterodimers [14]. Therefore, afatinib represents an ErbB family blocker that covalently binds to, and irreversibly blocks, signaling from all possible ErbB homodimers and heterodimers [13,15]. It inhibits EGFR more potently than first-generation EGFR TKIs and has greater anti-NSCLC preclinical activity, both in cell-based experiments and in animal models [15,16]. Afatinib has demonstrated PFS benefit versus chemotherapy in two Phase III trials, LUX-Lung 3 and LUX-Lung $6[17,18]$. Also, in contrast to first-generation EGFR TKIs, afatinib was associated with significant improvements in OS versus chemotherapy in both trials, based on a prespecified analysis of patients with the most common category of EGFR mutation, deletions within exon 19 (Del19) [19]. In contrast to first- and second-generation agents, third-generation EGFR-targeted TKIs such as osimertinib were designed to covalently bind to only some mutated forms of EGFR, thus lowering the incidence of EGFR wild-type mediated toxicities. While such agents were developed in a second-line setting, osimertinib has recently demonstrated a median PFS benefit of 17.7 months (independent assessment) in previously untreated patients with EGFR mutation-positive (Del19 or L858R) NSCLC [20], and is now approved in this setting.

While EGFR-targeted agents are associated with robust clinical benefits, acquired resistance to treatment is inevitable. The most common resistance mechanism to first- and second-generation EGFR-targeted TKIs is the so-called 'gatekeeper' T790M mutation in exon 20 of EGFR, which has been identified in around $50-70 \%$ of cases [21-25]. This mutation increases the affinity of EGFR for ATP, thus reducing the potency of ATP-competitive TKIs [26]. The identification of T790M provided the original rationale for the development of osimertinib and other third-generation TKIs, which also inhibit T790M-positive tumors. Indeed, second-line osimertinib has demonstrated striking activity in this setting and is associated with high response rates $(\sim 60-70 \%)$ and impressive PFS ( $\sim 10-12$ months) in T790M-positive patients $[23,27,28]$.

Several T790M-independent mechanisms of resistance to first-generation EGFR-TKIs have been identified including aberrations affecting other receptors (e.g., HER2, HER3, MET, RET and IGF-1 receptors) leading to compensatory signaling via intracellular pathways such as the PI3K/AKT/mTOR and JAK2/STAT3 cascades [29,30], transformation to small cell histology [22] and epithelial to mesenchymal transition [31]. Less data are available regarding T790M-independent resistance mechanisms to second-generation TKIs including afatinib. Unsurprisingly, given its mechanism of action, compensatory signaling via other ErbB family members has not been identified as a mechanism of resistance to afatinib. Rather, resistance mechanisms through non-target pathways such as MET amplification or histological transformation have been reported [32-34].

In contrast to first- and second-generation TKIs, no predominant mechanism of acquired resistance has been reported following first-line treatment with osimertinib, although few data are available at the moment. Resistance mechanisms identified to date appear to be highly heterogeneous [35,36]. In a cohort of 19 patients treated with first-line osimertinib, putative resistance mechanisms were only identified in nine cases and included mutations 
in MEK1, KRAS, PI3KCA, HER2 or JAK2 or amplification of KRAS, MET or EGFR. Two patients acquired the tertiary EGFR resistance mutation, C797S. Consequently, possible targeted treatment options following first-line osimertinib remain unclear. More data are available regarding resistance to osimertinib in later-line settings. In general, such resistance mechanisms seem to differ depending on whether tumors maintain or lose the T790M mutation [37]. Mechanisms include the accrual of C797S in approximately $20-40 \%$ of cases, transformation to small cell histology, amplification of HER2, MET or FGFR, and mutations in BRAF [37-40]. EGFR-mediated resistance occurs more frequently in cases where T790M is preserved [37]. In patients with T790M-positive tumors treated with second-line osimertinib, C797S appears to predominantly occur in cells with a background Del19 mutation and in cis with T790M (i.e., on the same allele [39,40]). Hence, combinations of currently available EGFR TKIs are not expected to be effective against triple EGFR mutants. However, such combinations could be effective in a first-line setting where preventing emergence of resistance is the major goal. Other EGFR mutations (e.g., L792X and L718Q) have also been associated with resistance to osimertinib [41]. In vitro studies indicate that L792X mutants may retain sensitivity to first- and second-generation EGFR TKIs when T790M is absent, whereas L718Q mutants are sensitive to afatinib but not gefitinib [42]. Such observations may be valuable for clinical practice.

Therefore, understanding the clonal evolution of tumors, in other words, the proliferation of certain cell types, with certain molecular characteristics, in response to the selective pressures conferred by treatment is of utmost importance. Ultimately, an understanding of how tumors develop at the cellular level in individual patients will be crucial in improving clinical outcomes by guiding rational therapeutic decisions with a view to delay resistance, and the identification of the most appropriate treatment options following the development of resistance. Recent progress with cutting-edge genomic technologies have demonstrated that $E G F R$ mutation-positive tumors can be complex and highly heterogeneous. Accordingly, there is a high risk of background genomic aberrations driving clonal expansion of a resistant, and potentially difficult-to-treat tumor, even if such aberrations are present in a very small number of tumor cells prior to initiation of therapy.

This review article comprises five sections. First, we briefly discuss recent developments in the general understanding of tumor clonality and the implications of clonal divergence on the diagnosis, clinical course and resistance to treatment of human cancers. Second, with these concepts in mind, we give an overview of recent findings that demonstrate considerable molecular complexity of EGFR mutation-positive NSCLC tumors and how this relates to clonal divergence, in other words, acquired resistance mechanisms, observed in patients with $E G F R$ mutation-positive NSCLC. Third, given the structural and mechanistic differences between first-, second- and third-generation EGFR-targeted TKIs, we compare and contrast the activity of these agents against cell models of EGFR mutation-positive tumor development, focusing on their activity against different EGFR mutations and cells with co-occurring genetic aberrations. Fourth, we discuss recent clinical data comparing different generations of EGFR-targeted TKIs. Finally, we discuss how emerging knowledge of resistance mechanisms over the course of tumor evolution may help drive the therapeutic sequencing of EGFR TKIs, with the aim of maximizing the time patients can remain chemotherapy-free and optimizing survival outcomes.

\section{The general implications of tumor heterogeneity on the diagnosis \& treatment of cancer}

It is generally accepted that tumors undergo complex Darwinian evolution over the course of their development, and during therapy [43]. This concept was first proposed in 1976 by Peter Nowell, who outlined a stepwise process by which tumor cell populations descended from a single progenitor or 'clone' acquire successive somatic mutations that confer a selective advantage [44]. Genomic instability of developing tumors creates a genetically diverse cell population that is subject to selective forces, as well as neutral changes over time (genetic drift) [45]. Eventually, selective outgrowth of clones that have a phenotypic advantage within a given microenvironment or therapeutic context results in the evolution of genetically distinct subclonal populations $[44,46]$.

While the concept of tumor evolution has been accepted for a long time, it is only with recent technological advances, such as next-generation sequencing (NGS) platforms, that it has been possible to dissect the development of tumors at sufficient resolution to enable the construction of divergence-based phylogenetic trees to illustrate tumor development, including NSCLC [47,48]. Moreover, new liquid biopsy technologies have facilitated the tracking of mutations over time to determine whether they are clonal or subclonal events [49,50]. This has allowed the definition of different categories of mutational events during the evolutionary process (Figure 1) [51]. 'Truncal' mutations are clonal mutations that are present in all tumor cells and regions, indicating a potential early role in driving oncogenesis. 'Branch' mutations are later subclonal events that are present in only a small proportion of 


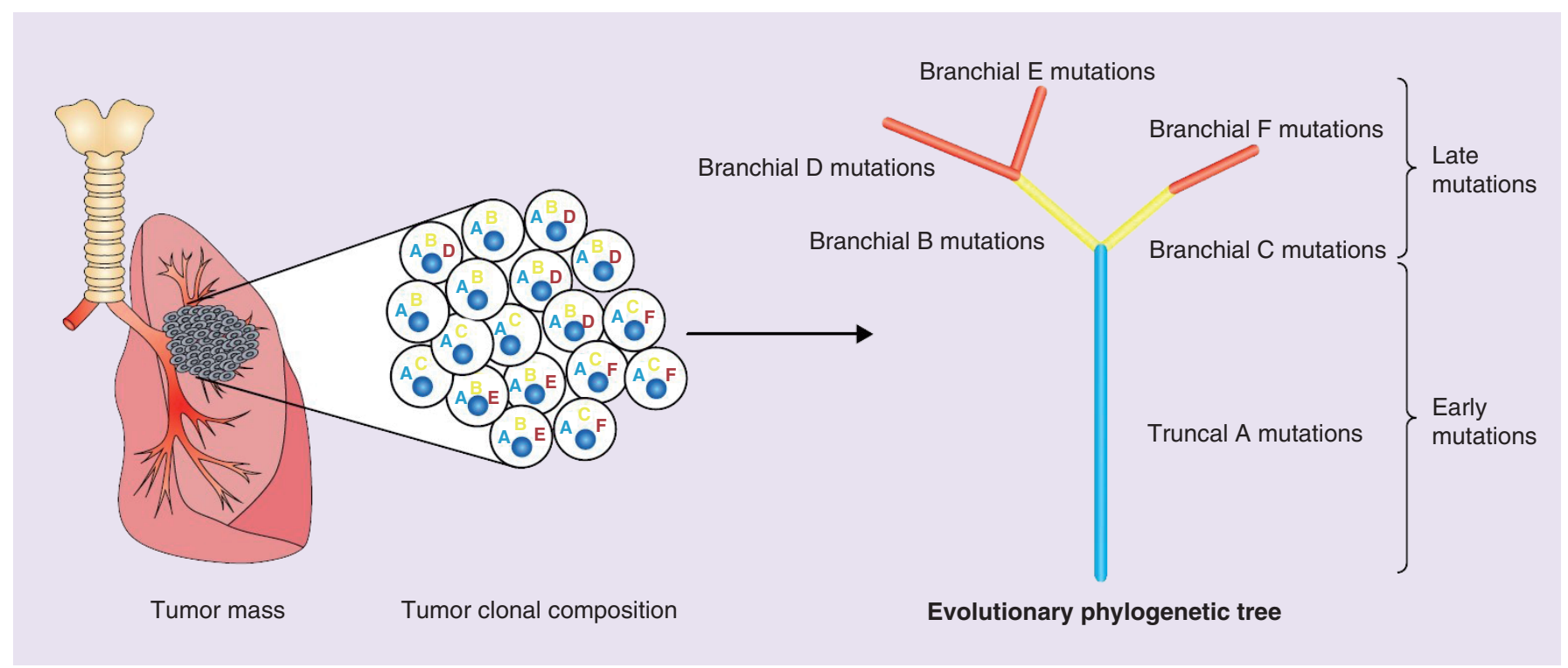

Figure 1. Phylogenetic tree analysis. Truncal mutations (A) occur early during tumorigenesis and are present in most tumor cells/regions. Branch mutations (B \& C) occur later in tumorigenesis and are present in some, but not all, tumor regions. Later branch mutations (D-F) tend to be highly localized within developing tumors.

Adapted with permission from [51] @ AACR (2015).

tumor cells and may emerge in subpopulations within discrete tumor locations (including in lung cancer), resulting in intratumoral clonal diversity $[43,48]$.

The clonal nature of tumor evolution underpins the difficulty of long-term treatment of tumors. Any treatment modality, be it traditional chemotherapeutics or targeted therapies, may impose strong selective pressure on tumor cells allowing resistant cells to survive which, under the right circumstances, may develop into a tumor which is resistant to treatment and, in terms of molecular characteristics, different to the original malignancy. This has obvious implications when considering treatment strategies. For example, 'truncal' mutations are often targets of established therapies, including those directed against EGFR mutations in NSCLC. Given the role of 'truncal' mutations as oncogenic drivers, such treatment is usually highly effective initially, but can drive the clonal development of 'branch' mutations. Indeed, substantial changes in clonal dominance over time have been observed in a number of human malignancies including NSCLC, breast cancer, multiple myeloma and acute lymphoblastic leukemia, whereby the mutational profile at disease progression or death can be markedly different to that at diagnosis $[43,46,49]$. The selective pressure of multiple lines of drug treatment can increase the complexity of tumors by facilitating the selection, and propagation, of cells that have intrinsic or acquired resistance to targeted treatment, thus influencing disease progression [51].

Given the impact of clonal evolution on the development of acquired resistance to treatment, it would be highly advantageous to understand the clonal architecture of tumors prior to initiation of, and during, therapy. In practice, however, this has proved difficult for technological and practical reasons. For example, subclonal populations may intermingle or be spatially separated within tumor lesions or between primary and metastatic sites, such that sampling bias can confound resolution of the clonal status of mutations. Therefore, a single biopsy in one tumor region might identify multiple subclones, while a biopsy in another region could give a false indication of clonality if dominated by an individual subclone. Of note, even in cases where one clone dominates the physical entity of a tumor, low frequency and often undetectable subclones can determine the clinical course and, depending on treatment sequence, affect therapy outcomes [49]. Indeed, multiple different subclones may harbor distinct resistance-conferring mutations rather than there being a single dominant resistance clone [43]. This spatial and temporal heterogeneity of tumors underpins the need for studies that use longitudinal tissue sampling. To this end, the use of 'liquid biopsies', analyzing cell-free (cf) DNA or circulating tumor cells in peripheral blood, provides a relatively noninvasive method for monitoring genomic alterations leading to disease progression [51]. However, such methodology will not provide any information on intra- or inter-tumor spatial heterogeneity. Liquid biopsy is a representation of the total tumor burden (primary and secondary tumors) and some tumors may shed 
DNA more than others. Furthermore, although cfDNA tests for the detection of activating and resistance $E G F R$ mutations have already entered clinical practice, further validation of the concept and sensitivity/specificity of such platforms is required. Concordance rates between paired plasma and tumor samples demonstrate high specificity but low sensitivity $(<50 \%)$, so false-negative results are possible [52]. Moreover, comparison of different platforms demonstrated concordance rates of 57-90\% for the detection of T790M [52]. This is consistent with experience of cfDNA tests in other tumor types [53], demonstrating that the congruence rates of commercially available tests can be suboptimal.

\section{The complexity of EGFR mutation-positive NSCLC tumors}

Emerging data indicate that the concepts described above are relevant to EGFR mutation-positive NSCLC tumors. Indeed, it is becoming increasingly clear that such tumors are genetically heterogeneous and undergo clonal evolution. Two sources of heterogeneity, which have possibly been underappreciated in the past, are: the level of co-occurring genetic alterations that are present in the background of EGFR mutation-positive tumors and the potential existence of several different types of specific EGFR mutation within a single tumor. Recent evidence uncovering these phenomena, and their likely clinical significance, are discussed below.

\section{Co-occurring genetic alterations}

Until recently, there were very few data regarding co-occurring genetic mutations in EGFR mutation-positive tumors, and how such mutations may evolve over the course of tumor growth, and in response to treatment with EGFR-targeted TKIs. This paucity of information was largely due to technological limitations that precluded the possibility of longitudinal genetic analysis over multiple time points. Initial large-scale sequencing analysis of NSCLC tumors focused on a single time-point (i.e., at tumor biopsy) at an early stage of tumor detection prior to initiation of treatment [54]. Such analysis indicated that EGFR mutations are probably early clonal events that drive oncogenesis. However, the potential role of co-occurring aberrations in driving tumor progression and treatment resistance remained an open question. These questions are now being addressed, fostered by the development of validated blood-based cfDNA assays. In a recent study, Blakely et al. undertook comprehensive genomic analysis of 68 relevant cancer genes using a validated cfDNA exome platform on 1122 consecutive DNA samples from 1006 patients with advanced stage EGFR mutation-positive NSCLC [49]. This analysis highlighted that additional genetic mutations are very common in late-stage tumors. In addition to $E G F R$, there was an average of $2.6 \pm 1.7$ co-occurring genetic alterations in the 68 cancer relevant genes in the samples tested; $93 \%$ of patients had at least one variant of known or likely functional importance. These additional aberrations included known oncogenic drivers in intracellular signaling and cell cycle genes such as PIK3CA, BRAF, MET, MYC, CDK6 and CTNNB1. The analysis also indicated that co-occurring mutations are very common in T790M-positive tumors. In 440 T790M-positive cases, there was an average of $2.4 \pm 1.9$ co-occurring genetic alterations in the cancer relevant genes. Frequent alterations were observed in the cell cycle pathway genes (24.1\%), DNA repair genes (10.9\%), epigenetic pathway genes (14.1\%), WNT pathway genes (15.5\%) and hormone signaling genes (6.8\%).

As expected, activating EGFR mutations were generally truncal (in $\sim 90 \%$ of samples) in the Blakely $e$ al. study, whereas T790M was more frequently restricted to branches ( $230 \%$ of samples) [49]. Approximately $40 \%$ of both Del19/L858R and T790M-positive tumors harbored additional branchial genetic aberrations, indicating that additional mutations evolve and develop over the course of the disease. Moreover, notwithstanding the limitations of liquid biopsies (which cannot detect spatial inter- or intra-tumor heterogeneity), data from patients who underwent longitudinal cfDNA analysis indicate that the genetic complexity of tumors probably increases over the course of treatment. In the 97 patients for whom longitudinal cfDNA, clinical context and treatment response data were all available, the frequency of additional somatic alterations increased with each line of therapy. Furthermore, the mean number of functional alterations was lower in patients who responded to EGFR-targeted TKI treatment (of any generation) than in patients who did not respond, possibly indicating lower tumor mutational burden in these patients [49]. These data indicate that EGFR mutation-positive tumors actually evolve to become more genetically complex during treatment and this complexity may be associated with primary and acquired resistance to successive lines of therapy. Therefore, understanding the baseline molecular makeup of the tumor and the mechanisms of resistance to particular agents may help drive clinical decision making in terms of selection of the most appropriate agents and sequence of therapies.

While the identification of T790M as the predominant mechanism of acquired resistance, and the development of third-generation EGFR TKIs, such as osimertinib, to target this aberration, were major advances in the treatment 
of EGFR mutation-positive NSCLC, it is open to debate how, and when, this aberration evolves during first-line treatment with first- or second-generation EGFR TKIs. It is unclear whether small numbers of T790M-positive cells pre-exist within the tumor prior to treatment initiation or, alternatively, whether T790M arises as a de novo branching event during treatment. This is an important question because the level of clonality of T790M at the point of acquired resistance may have implications in terms of clinical response, and the duration of response, to third-generation EGFR TKIs, in other words, the greater the uniformity of T790M-driven resistance at progression, the higher the likelihood of enduring response to agents that specifically target this aberration. Again, this question has proved to be very difficult to directly address. Even highly sensitive technologies, including NGS and dropletdigital PCR may not have the resolution to detect rare pre-existing T790M-positive clones at the cellular level [50]. While several studies have reported the detection of low-frequency T790M clones in tumors prior to the initiation of first-line therapy [55,56], doubts have been raised regarding the validity of these findings due to the possibility of sequencing artifacts related to tissue fixation methodology [57].

Recent analysis of EGFR mutation-positive cell lines provided compelling evidence that T790M can arise from pre-existing clones prior to the initiation of first-line therapy, but can also develop during the course of treatment from a pool of drug-resistant 'persister' cells [58]. These cells have been observed in several studies [59-61], and survive treatment, possibly due to epigenetic adaptations, but do not expand until they acquire additional resistance mechanisms. Hata et al. undertook a series of experiments where EGFR mutation-positive cells were cultured in the presence of escalating concentrations of gefitinib [50]. Two different categories of resistant clones developed; those that displayed early resistance (i.e., within 6 weeks) and those that emerged later (within 24 weeks). All early resistant clones were T790M-positive, and by utilizing a recently developed DNA barcoding technology (ClonTracer) [62], it was demonstrated that these clones were derived from pre-existing T790M-positive cells. In contrast, late resistant clones were initially T790M-negative; T790M arose in drug-tolerant persister cells, and gradually expanded to become the dominant clone. Resistant cells that remained T790M negative had a plethora of other mutations at low allele frequencies including mutations in NRAS, KRAS, BRAF and RET. These findings indicate that T790M may gradually evolve from de novo events occurring within persister cells, within a background of complex genetic heterogeneity. Of note, late resistant clones appeared to be less sensitive to third-generation EGFR TKIs than early resistance clones, presumably reflecting the distinct evolutionary paths by which T790M arose. These preclinical observations have clinical ramifications and suggest that first-line treatment approaches that target the predominant oncogenic driver (i.e., EGFR activating mutations) but also limit the development of persister cells, with complex mutational profiles, would be beneficial, as these cells appear to develop in difficult-to-treat tumors.

\section{EGFR mutation heterogeneity}

The most frequently identified EGFR mutation category are Del19 mutations ( $\sim 45 \%$ of all EGFR mutations), which represent at least 30 variants. The next most prevalent mutation is the L858R point mutation in exon 21 ( $\sim 40 \%$ of EGFR mutations). Together Del19 and L858R are termed 'common' mutations, which are known to be highly sensitive to all generations of EGFR-targeted TKIs, although collectively Del19 mutations are typically regarded as being more sensitive than L858R [63]. Recent improvements in detection methods have indicated that 'uncommon' EGFR mutations are more prevalent than previously thought. According to the catalogue of somatic mutations in cancer (COSMIC) database, in 2016, 594 types of EGFR mutation had been reported [64]. Categories of uncommon mutation include insertions in exon 19 (Ins19; 0.6\% of all EGFR mutations) or 20 (Ins20; 6\%), as well as point mutations in exon 18 at position G719 (G719X; 3\%), exon 21 L861Q mutations ( $1 \%$ ) and exon 20 S768I mutations ( $\sim 1 \%$ of EGFR mutations) [64]. Despite being present in at least $10 \%$ of patients with $E G F R$ mutation-positive NSCLC [65], most Phase III trials excluded patients with 'uncommon' mutations (LUX-Lung 3 and 6 being notable exceptions).

In addition, it is becoming increasingly clear that some patients with EGFR mutation-positive NSCLC have tumors that harbor more than one type of $E G F R$ mutation, predominantly in cis, in other words, within the same $E G F R$ allele (so-called 'compound' mutations) [64,66]. Compound mutations are associated with poorer clinical outcomes compared with simple mutations [67]. Recent NGS analysis indicates that compound mutations are present in up to $25 \%$ of patients with EGFR mutation-positive NSCLC [66,67]. Most commonly, compound mutations consist of an EGFR TKI-sensitizing mutation such as Del19, L858R or G719X, together with a rare mutation of unknown clinical significance [68]. A minority of compound mutations comprise only uncommon atypical mutations $[66,67,69]$. 
Sequencing of EGFR Del19
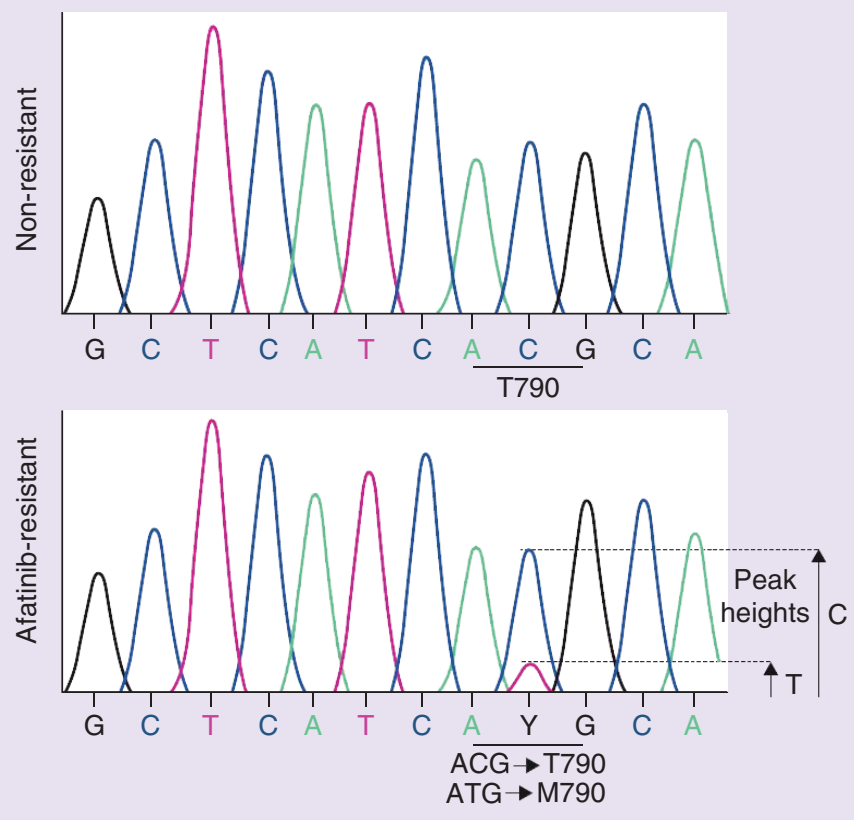

T790M gene dosage

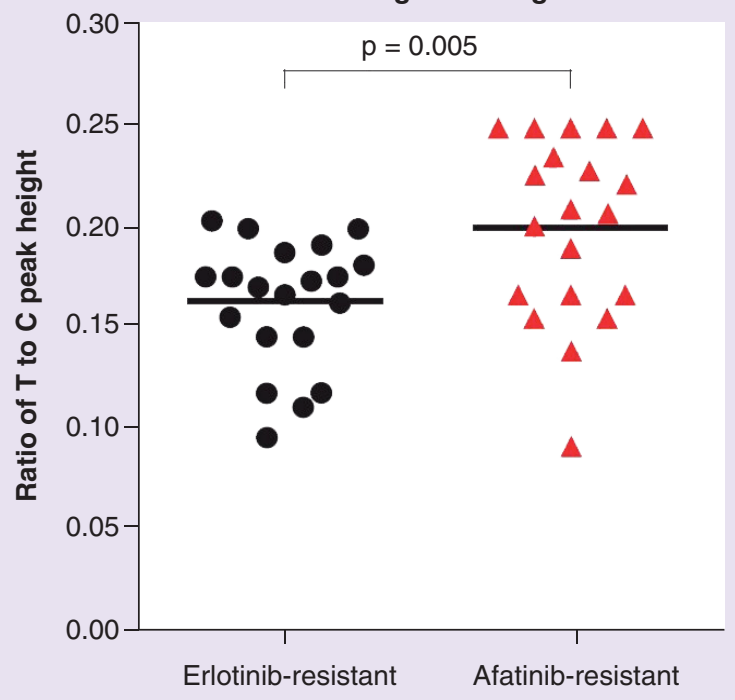

Figure 2. T790M gene dosage in Ba/F3 EGFR del19 cells resistant to erlotinib and afatinib. Ba/F3 cells expressing EGFR del19 were mutagenized with N-ethyl-N-nitrosourea and subsequently treated with erlotinib or afatinib to isolate drug-resistant clones. The EGFR sequence at residue T790 was analyzed in 20 wells displaying erlotinib- or afatinib-resistant cell growth by DNA extraction followed by PCR and Sanger sequencing (chromatograms, left). The peak heights of $C$ and $T$ at codon position 2 of T790 were calculated for each drug-resistant well and plotted (graph, right). Bar, mean and p-value, unpaired t-test.

\section{Preclinical differences in activity of EGFR-directed TKIs against NSCLC tumor cells} Cells with co-occurring genetic alterations

Ideally, given the high prevalence of co-occurring genetic alterations in EGFR mutation-positive NSCLC, TKIs should have a broad inhibitory profile to mitigate for the heterogeneity of tumors and the risk of rapid expansion of resistant subclones. Also, if T790M-mediated acquired resistance occurs, it would be advantageous that the T790M clone is as homogeneous as possible to maximize the chances of a prolonged response to third-generation TKIs like osimertinib. Furthermore, low levels of T790M clonality may compromise the ability of tissue or liquid assays to detect the mutation.

As co-occurring aberrations in EGFR mutation-positive tumors are highly heterogeneous, and usually occur in only a small number of cells, it is technologically very difficult to compare the activity of EGFR-directed TKIs against them. Nevertheless, we have undertaken experiments that have specifically assessed the clonality of tumor cells following acquired resistance. We cultured Ba/F3 cells that expressed Del19 EGFR. The cells were subjected to $\mathrm{N}$-ethyl-N-nitrosourea mutagenesis and cultured in the presence of afatinib or erlotinib (50-fold $\mathrm{IC}_{50}$ concentration) for 3 weeks. Resistant wells were selected and genomic DNA was extracted. Subsequently, the tyrosine kinase domain of EGFR was amplified by PCR and sequenced. The majority of resistant wells for both afatinib and erlotinib were T790M-positive. Interestingly, the frequency of T790M alleles was higher in afatinib-treated cells than erlotinib-treated cells as determined by measuring sequencing peak heights, indicating that T790M clones were more homogeneous following afatinib treatment (Figure 2). This observation possibly suggests that afatinib, due to its broader inhibitory profile, is active against a greater proportion of co-occurring aberrations than erlotinib in Del19 cells, preventing their clonal expansion. Another possibility is that, due to its modest activity against T790M, a higher allele frequency of T790M is required to generate resistance to afatinib compared with erlotinib or gefitinib. These inferences are speculative and require further investigation. 


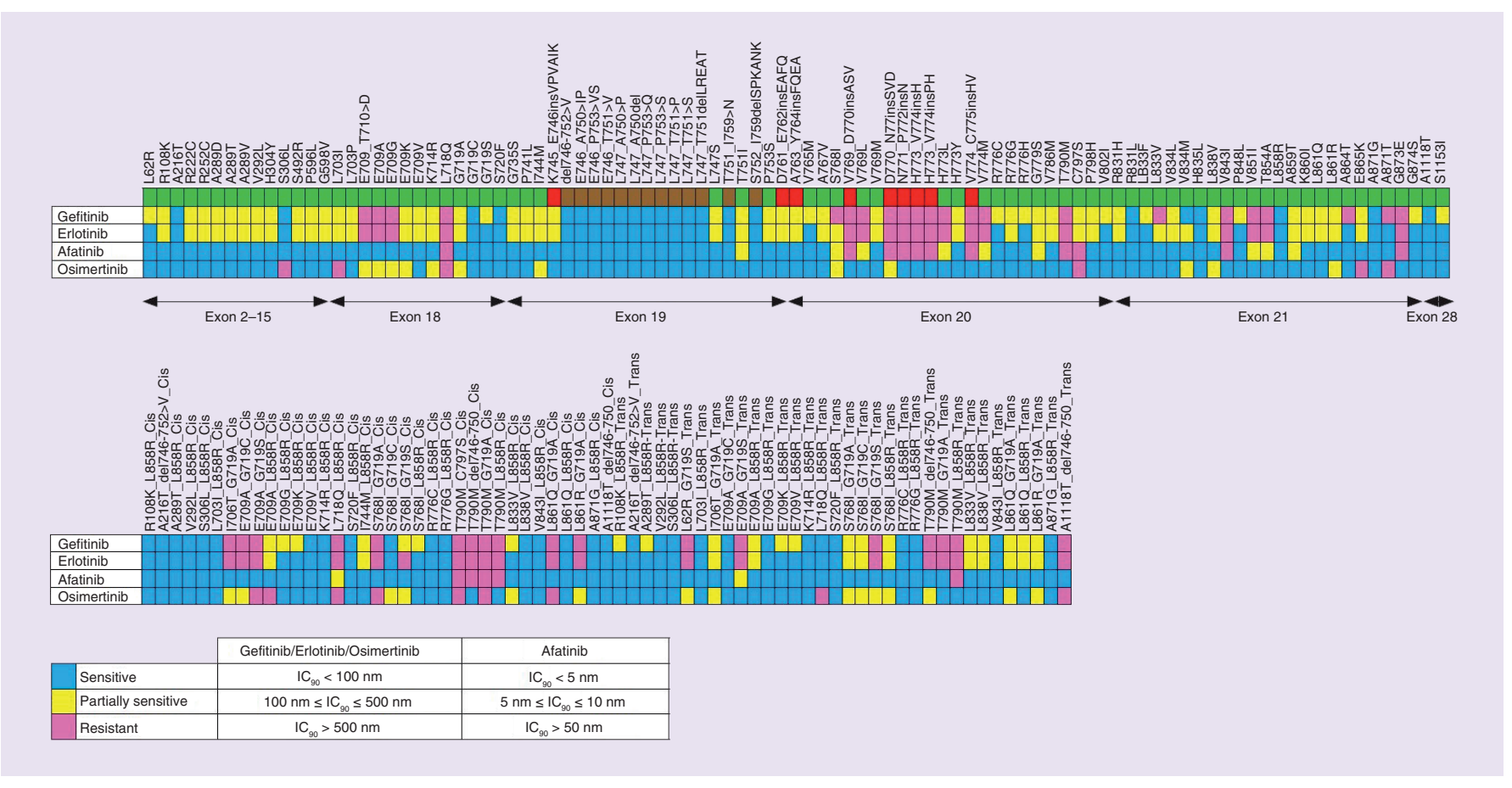

Figure 3. Assessment of drug sensitivity of uncommon single and compound EGFR mutations using the mixed-all-nominated-mutants-in-one (MANO) method in Ba/F3 cells.

Brown: Deletion; green: Missense mutation; red: Insertion.

Adapted with permission from [66] (CAAAS (2017).

\section{Cells with uncommon or compound EGFR mutations}

The heterogeneity of EGFR mutations in EGFR mutation-positive NSCLC has potential clinical implications because different EGFR-targeted TKIs can have different activities against specific mutations. Therefore, treatment with EGFR TKIs that have limited efficacy against certain mutations may drive the clonal expansion of those insensitive mutations. Moreover, it is possible that exposure to drugs with a limited spectrum of activity against different mutations within a single oncogene may actually drive the selection of cells with compound mutations, leading to the development of difficult-to-treat resistant tumors. This phenomenon has been identified in patients with BCR-ABL1 mutation-positive chronic myeloid leukemia [70]. It may be important, therefore, that EGFR TKIs have as broad as an inhibitory profile as possible in terms of activity against specific EGFR mutations.

Emerging preclinical evidence indicates that second-generation TKIs may have a broader inhibitory profile against EGFR mutations than first- or third-generation TKIs [64,66]. In a recent study, Kobayashi and Mitsudomi collated available preclinical data assessing the activity of first-generation (gefitinib and erlotinib), second-generation (afatinib, dacomitinib, neratinib) and third-generation TKIs (osimertinib, rociletinib) in Ba/F3 cells expressing specific EGFR mutations [59]. This analysis demonstrated that second-generation TKIs have a broad inhibitory profile with afatinib being particularly active against exon 18 mutations, Del19 mutations, Ins19 mutations and S768I.

Another recent preclinical study assessed the activity of different EGFR-directed TKIs against uncommon $E G F R$ variants of unknown significance. By utilizing a high-throughput functional assay in $\mathrm{Ba} / \mathrm{F} 3$ cells, Kohsaka et al. evaluated the sensitivity of over a hundred different variants of unknown significance, including compound mutations [66]. Interestingly, afatinib showed activity against almost all mutations tested and, in many cases, was more potent than erlotinib and gefitinib (Figure 3). Afatinib also showed a broader spectrum of activity against uncommon $E G F R$ variants compared with osimertinib, although, as expected, afatinib was less effective against T790M than osimertinib [66].

A further interesting finding from the Kohsaka $e t a l$. study was that the emergence of certain compound mutations (e.g., L858R and E709A/G) was associated with diminished response to gefinitib and erlotinib. However, the sensitivity of L858R_E709 compound mutations to afatinib was not remarkably different to that observed for 
L858R alone $\left(\mathrm{IC}_{50}<0.1 \mathrm{nM}\right)$. Indeed, afatinib reduced the viability of cells expressing numerous compound mutations, including those involving Del19, G719X and/or S768I mutations (Figure 3); only compound mutations involving T790M were considered to be resistant to afatinib [66]. Of note, cDNA-based amplicon sequencing and droplet digital PCR indicated that many compound mutations detected occurred in cis. This means that the co-existing mutations are likely to have an additive impact on the tertiary structure of EGFR, which could underlie the relevance of compound mutations in terms of acquired resistance. Importantly, preclinical data described above indicate that afatinib, possibly due to its alternative mechanism of action, may reduce the selection for compound mutations as a mechanism of acquired resistance compared with gefitinib, erlotinib or osimertinib.

\section{Clinical data comparing different generations of EGFR-targeted TKIs Head-to-head clinical studies}

While there is a plethora of clinical data validating the first-line use of EGFR-targeted TKIs in patients with EGFR mutation-positive NSCLC, until recently, there have been very few head-to-head comparisons between the different generations of agent. However, in the last few years, several trials (LUX-Lung 7, FLAURA, ARCHER-1050) have demonstrated that second- and third-generation TKIs are more efficacious than first-generation TKIs in a front-line treatment setting.

LUX-Lung 7 was a global Phase IIb trial that compared afatinib $(\mathrm{n}=160)$ versus gefitinib $(\mathrm{n}=159)$ in patients with EGFR mutation-positive (Del19 or L858R) NSCLC [71]. The study had three co-primary end points; PFS, OS and time to treatment failure (TTF). TTF was chosen as an end point to reflect the fact that treatment with TKIs is often continued beyond radiological progression in 'real-world' clinical practice. Both PFS (median: 11.0 vs 10.9 months; hazard ratio [HR]: 0.73 [95\% CI: 0.57-0.95], $\mathrm{p}=0.017$ ) and TTF (median 13.7 vs 11.5 months; HR: 0.73 [95\% CI: $0.58-0.92], \mathrm{p}=0.0073$ ) were significantly improved with afatinib compared with gefitinib. There was a trend toward improved OS (median: 27.9 vs 24.5 months; HR: 0.86 [95\% CI: 0.66-1.12], $\mathrm{p}=0.2580$ ) [72]. The response rate with afatinib was higher than with gefitinib (70 vs 56\%; odds ratio: 1.87 [95\% CI: 1.18-2.99], $\mathrm{p}=0.0083)$ [71]. Similar response rates were observed in patients with Del19 mutations (73\% with afatinib and $66 \%$ with gefitinib). However, in patients with tumors harboring L858R mutations, the response rate was 66\% with afatinib versus $42 \%$ with gefitinib.

Given its limited availability at the time of the study, only 50 patients went on to receive osimertinib following afatinib $(n=27)$ or gefitinib $(n=23)$. However, clinical outcomes in the patients were encouraging. At the time of analysis, median OS had not been reached in either group. A further analysis, including patients from LUX-Lung 3 and LUX-Lung 6 who received sequential afatinib and osimertinib $(n=10)$, demonstrated prolonged duration of therapy with osimertinib (median: 20.2 months) [73].

The recent Phase III ARCHER-1050 trial compared dacomitinib $(\mathrm{n}=227)$, another ErbB family blocker, to gefitinib $(\mathrm{n}=225)$ in patients with EGFR mutation-positive (Del19 or L858R) NSCLC [74]. Although there were differences in trial populations between LUX-Lung 7 and ARCHER-1050 (e.g., patients with stable brain metastases were included in the former but excluded in the latter), ARCHER-1050 provided further evidence that second-generation EGFR-directed TKIs are more efficacious than first-generation TKIs. Dacomitinib significantly prolonged PFS compared with gefitinib (14.7 vs 9.2 months; HR: 0.59 [95\% CI: 0.47-0.74], p < 0.0001). Notably, it also significantly improved OS versus gefitinib (median: 34.1 vs 26.8 months; HR: 0.76 [95\% CI: $0.58-0.99], p=0.044$ [75]. The response rate was $75 \%$ with dacomitinib and $72 \%$ with gefitinib. Response rates were similar regardless of mutation type. The duration of response was longer with dacomitinib than gefitinib (median: 14.8 vs 8.3 months; HR: 0.40 [95\% CI: 0.31-0.53], $\mathrm{p}<0.0001$ ).

Osimertinib has also demonstrated superior clinical benefits versus first-generation EGFR TKIs. The Phase III FLAURA trial compared osimertinib $(\mathrm{n}=279)$ versus gefitinib or erlotinib $(\mathrm{n}=277)$ in patients with EGFR mutation-positive (Del19 or L858R) NSCLC [20]. PFS was significantly improved in the osimertinib arm (by investigator assessment, median: 18.9 vs 10.2 months; HR: 0.46 [95\% CI: 0.37-0.57], p < 0.001). Response rate was similar between treatment arms ( $80 \%$ with osimertinib and $76 \%$ with erlotinib/gefitinib) but duration of response was higher with osimertinib (17.2 vs 8.5 months). OS data were immature at the time of publication $(25 \%)$. The crossover rate to osimertinib for patients discontinuing study treatment in the erlotinib/gefitinib arm was only $23 \%$. Of further note, afatinib was not included in the comparator arm of FLAURA, so while the outcome data are very encouraging, it is not possible to draw any conclusions regarding the relative efficacy of second- and third-generation EGFR-directed TKIs based on FLAURA. 


\section{Activity against uncommon EGFR mutations}

Clinical data on the activity of EGFR-targeted agents against uncommon mutations are limited. The general consensus in the literature indicates that outcomes are generally unfavorable in patients with tumors harboring uncommon mutations [19,76,77]. Post-hoc analysis of the Phase III NEJ002 trial demonstrated that OS with gefitinib was significantly shorter in patients with uncommon EGFR mutations (G719X or L861Q; $\mathrm{n}=10$ ) versus those with common mutations (median: 11.9 vs 29.3 months; $p<0.001$ ) [78]. Other clinical studies are in line with the observations in NEJ002 and demonstrated poor outcomes with first-generation EGFR TKIs in patients with uncommon mutations [76,77]. Furthermore, the outcomes appear to be heterogeneous depending on the type of uncommon mutation. Certain mutations, for example, exon 18 G719X mutations ( $n=10)$, exon 20 S768I ( $n=6)$ and exon $19 \mathrm{~K} 757 \mathrm{R}(\mathrm{n}=1)$ and E746G $(\mathrm{n}=1)$ mutations all appeared to confer a good outcome with erlotinib treatment, whereas exon 18 S720 mutations $(n=3)$ was associated with a particularly poor outcome [77].

Available clinical data with afatinib in patients with uncommon EGFR mutations appear to corroborate preclinical findings of broad activity. A post-hoc analysis of the LUX-Lung 2, 3 and 6 trials identified 75 afatinib-treated patients with tumors harboring uncommon mutations, including G719X ( $\mathrm{n}=18), \mathrm{L} 861 \mathrm{Q}(\mathrm{n}=16)$ and S768I $(\mathrm{n}=8)$ single/compound mutations [79]. In this analysis, median PFS among 38 patients with uncommon mutations/duplications in exons 18-21 was 10.7 months (95\% CI: 5.6-14.7) and median OS was 19.4 months (95\% CI: 16.4-26.9). Based on these findings, the US FDA recently expanded the front-line indication for afatinib to include NSCLC harboring uncommon EGFR alterations in L861Q, G719X and/or S768I. Outcomes in patients with Ins20 mutations ( $\mathrm{n}=23$ ) were poor (PFS 2.7 months; 95\% CI: 1.8-4.2), reflecting the observation that while afatinib had moderate in vitro activity against these mutations, sufficient inhibition was not achieved in patients with standard dosing.

Additional evidence for afatinib's clinical activity against uncommon EGFR mutations comes from a Phase IIIb single-arm open-label study (1200.66; NCT01953913) which included 67 TKI-naive patients with NSCLC tumors harboring uncommon (Ins20, L861Q; G719S/A/C, T790M, S768I) mutations; median PFS in these patients was 9.1 months (95\% CI: 5.6-13.6) [80]. Finally, an analysis of 165 patients with EGFR mutation-positive NSCLC who received first-line afatinib in real-world practice in South Korea showed that tumors harboring uncommon mutations other than T790M were sensitive to afatinib. Median PFS was not reached for those with uncommon mutations excluding T790M $(\mathrm{n}=10)$ versus 4.7 months in those with de novo T790M ( $\mathrm{n}=4 ; \mathrm{p}=0.01)$; median PFS in patients with Del19- and L858R-positive tumors was 19.1 and 15.1 months, respectively [81]. The potential advantages of afatinib versus erlotinib in compound mutations have also been recognized clinically. In a case study of a patient with a compound L858M/L861Q in cis mutation, treatment with afatinib overcome primary resistance to erlotinib [82].

\section{Could differences in clinical activity with EGFR TKIs reflect differences in the impact on clonal evolution?}

We speculate that the results from LUX-Lung 7, ARCHER-1050 and FLAURA could potentially be explained from a clonal evolution perspective. As discussed, EGFR mutation-positive tumors can be highly heterogeneous. Therefore, the broader inhibitory profile of second-generation TKIs against other ErbB family receptors or particular mutants could prevent emergence of tumor cells that would otherwise survive treatment with first-generation TKIs (Figure 4). This, in turn, could delay the onset of clonal expansion and acquired resistance to therapy, which is possibly reflected in the observed improvements in PFS with afatinib and dacomitinib versus the first-generation TKI, gefitinib. Moreover, the encouraging clinical benefit with sequential afatinib and osimertinib observed in recent retrospective studies $[73,83,84]$, could indicate that resistant T790M-positive clones are more homogeneous than those that develop during treatment with first-generation TKIs (Figure 4). For example, one study demonstrated that response rates and PFS were significantly better in patients who received sequential afatinib and osimertinib than patients who received sequential gefitinib/erlotinib and osimertinib [84]. This hypothesis is supported by our preclinical data and warrants further investigation. The impressive clinical benefits with osimertinib in FLAURA probably reflect its strong inhibitory activity against T790M, the major resistance mechanism to first- and secondgeneration EGFR TKIs, thus preventing the clonal expansion of pre-existing T790M cells or the development of de novo T790M-positive clones. Indeed available evidence indicates that de novo T790M mutations do not occur in patients treated with first-line osimertinib [36]. As previously mentioned, it appears that acquired resistance mechanisms to first-line osimertinib are heterogeneous, though data are limited [36]. More genomic data are required 


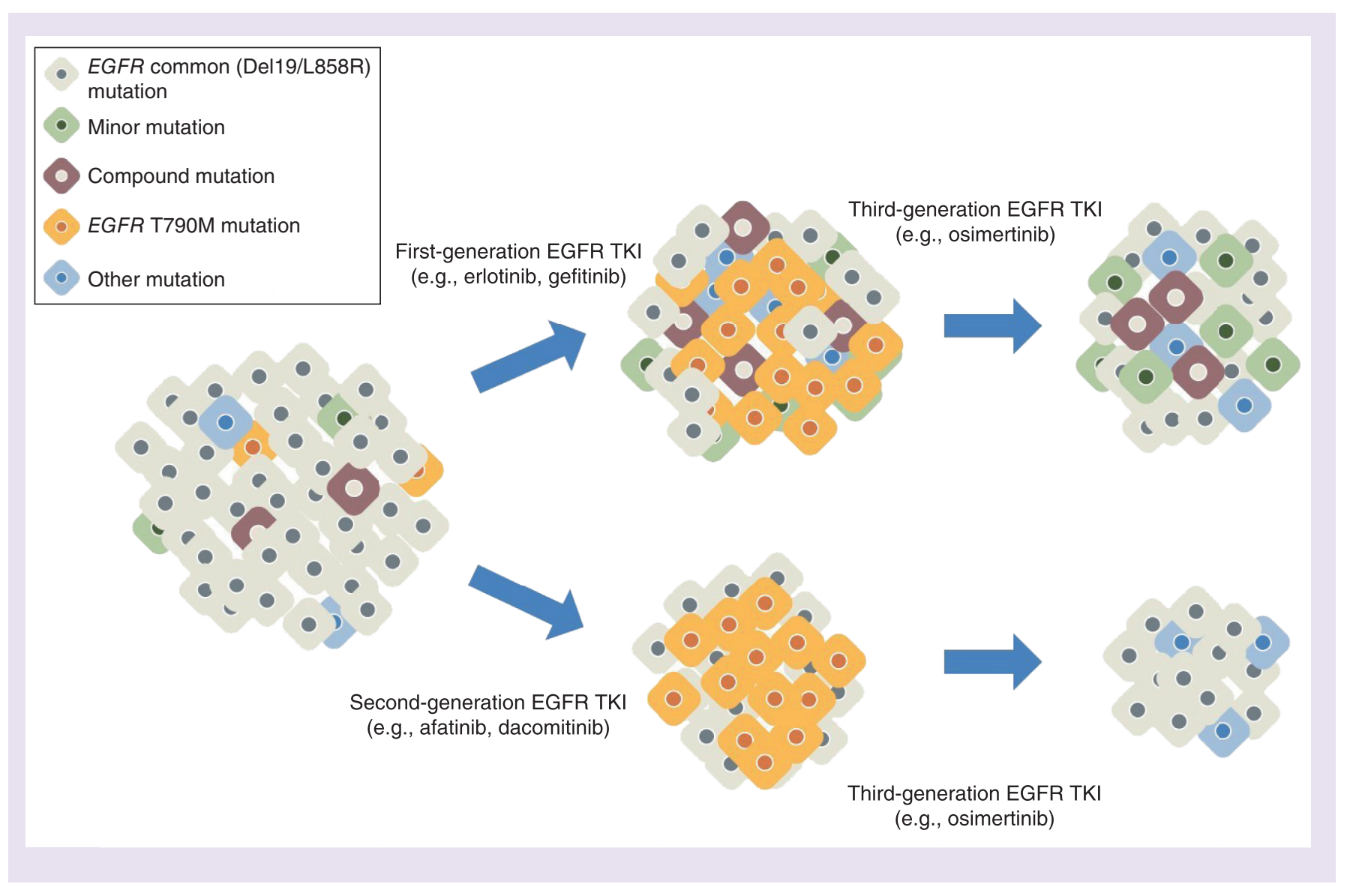

Figure 4. Hypothetical changes in clonal composition over time of non-small-cell lung cancer, tumors treated with sequential EGFR tyrosine kinase inhibitors.

TKI: Tyrosine kinase inhibitor.

to help define potential drug combinations, and drive the development of additional agents, that may be active in the post-osimertinib setting.

\section{Conclusion \& future perspective}

The availability of several EGFR-targeted agents has revolutionized treatment for patients with EGFR mutationpositive NSCLC. However, these developments pose complex questions in terms of how best to utilize available agents, and how to sequence therapy to maximize clinical benefit and prolong the time patients remain free from chemotherapy. Clearly, these are multifaceted questions and depend on a number of factors including first-line efficacy and tolerability of different agents, activity against specific EGFR alleles, impact on quality of life, physician experience and preference as well as cost and reimbursement considerations. A key factor, however, is which treatment regimen is most likely to maximize OS, which in turn depends on the availability of subsequent therapies beyond first-line EGFR-directed TKIs. A greater understanding of the clonality of EGFR mutation-positive tumors, and how tumors evolve in response to different agents, is integral to address this question.

Head-to-head clinical trials clearly demonstrate the superiority of second- and third-generation TKIs compared with first-generation TKIs in a first-line treatment setting. However, second- and third-generation TKIs have not been directly compared. Therefore, the optimal first-line treatment for patients with EGFR mutation-positive NSCLC remains an open question, especially given the current paucity of mature OS data with different agents. We hypothesize that broad ErbB family inhibition could be a preferable first-line treatment choice given emerging knowledge about the heterogeneity and clonal evolution of EGFR mutation-positive tumors. Moreover, given the preponderance of T790M after first- and second-generation EGFR TKIs, in principle, more than half of all patients with EGFR mutation-positive NSCLC could be eligible for, and benefit from, second-line osimertinib. In 
this regard, one needs, of course, to acknowledge the challenge of a second biopsy for the patient, the technological and conceptual limitations of liquid biopsies, and the limited availability of sensitive test platforms, like NGS, in 'real-world' clinical practice [85].

While data from the recent FLAURA trial are encouraging and position osimertinib as a first-line treatment option in patients with EGFR mutation-positive NSCLC [20], targeted treatment options following osimertinib resistance remain poorly defined at this time because of the heterogeneity of resistance mechanisms [36]. Therefore, at the moment, the most likely treatment option beyond osimertinib is chemotherapy. Accordingly, further clinical trial data are needed to assess optimal sequence of therapy with EGFR TKIs in individual patients over the course of their treatment, with OS as the key end point. Detailed analysis of the changing molecular features of tumors, as they continually evolve in response to treatment, will be a fundamental aspect of future clinical studies [86]. This will require further improvements, and better access to, molecular genomic technologies. Ultimately, monitoring of tumor clonality will help drive rational treatment choices with the ambition of turning $E G F R$ mutation-positive NSCLC into a chronic disease in many patients.

\section{Executive summary}

The general implications of tumor heterogeneity on the diagnosis \& treatment of cancer

- Tumors evolve over the course of their development, and during therapy. Genomic instability of developing tumors creates a genetically diverse cell population that is subject to selective forces; clones that have a phenotypic advantage within a given microenvironment or therapeutic context result in the evolution of genetically distinct subclonal populations.

- Clonal evolution plays a key role in acquired resistance of tumors to treatment. Therefore, it would be highly advantageous to understand the clonal architecture of tumors prior to initiation of, and during, therapy. Despite technological advances, for example, next-generation sequencing and liquid biopsy techniques, this still represents a major challenge.

The complexity of EGFR mutation-positive non-small-cell lung cancer tumors

- It is becoming increasingly clear that EGFR mutation-positive non-small-cell lung cancer (NSCLC) tumors are genetically heterogeneous and undergo clonal evolution. Two sources of heterogeneity are: co-occurring genetic alterations within other genes that are present in the background of EGFR mutation-positive tumors and different EGFR mutations within a single tumor.

Preclinical differences in activity of EGFR-directed tyrosine kinase inhibitors against NSCLC tumor cells

- Approved first- (erlotinib, gefitinib), second- (afatinib) and third-generation (osimertinib) tyrosine kinase inhibitors (TKIs) are available for the first-line treatment of EGFR mutation-positive NSCLC. These agents have different pharmacological profiles: erlotinib and gefitinib are EGFR-selective reversible inhibitors; afatinib is an irreversible ErbB family blocker; osimertinib is an EGFR selective wild-type sparing inhibitor. Owing to differences in pharmacological profile, different EGFR TKIs are likely to confer different selective pressures on developing EGFR mutated tumors.

- Compared with first-generation EGFR inhibitors, afatinib (and possibly dacomitinib), besides the broader ErbB inhibitory spectrum, is also active against several EGFR mutations, including compound mutations and uncommon EGFR mutations. This broad inhibitory profile may help delay the expansion of particular resistant subclones, resulting in more homogeneous subclones with high T790M gene dosage.

Clinical data comparing different generations of EGFR-targeted TKIs

- Recent head-to-head clinical trials (LUX-Lung-7, ARCHER-1050) have demonstrated that second-generation EGFR TKIs are superior to first-generation EGFR TKIs in patients with EGFR mutation-positive NSCLC. The recent FLAURA trial demonstrated that osimertinib is superior to first-generation EGFR TKIs; there are no prospective data comparing second- and third-generation EGFR TKIs. Differences in clinical activity could reflect differences in the impact of clonal evolution of tumors.

Conclusion \& future perspective

- At present, the optimal use of EGFR TKIs has not been defined. In the clinical setting, T790M-positive clones represent more than half of the resistant clones after erlotinib or afatinib treatment; in the preclinical setting, our data suggest that afatinib-resistant subclones are relatively homogeneous, display high T790M gene dosage and are likely to be very sensitive to second-line osimertinib.

- Emerging, albeit, limited clinical data have shown encouraging survival outcomes in patients treated with sequential afatinib/osimertinib.

- In the future, further technological developments, and the monitoring of the evolution of tumors during treatment, will be instrumental in guiding the optimal treatment strategy of EGFR mutation-positive NSCLC patients. 


\section{Author contributions}

The authors were fully responsible for all content and editorial decisions, were involved at all stages of manuscript development and have approved the final version.

\section{Financial \& competing interests disclosure}

S Kohsaka has received research support from AstraZeneca and Boehringer Ingelheim. F Solca and M Petronczki are employees of Boehringer Ingelheim. M Maemondo has received honoraria from Chugai Pharmaceutical, AstraZeneca and Boehringer Ingelheim. The authors have no other relevant affiliations or financial involvement with any organization or entity with a financial interest in or financial conflict with the subject matter or materials discussed in the manuscript apart from those disclosed.

Medical writing assistance, supported financially by Boehringer Ingelheim, was provided by Lynn Pritchard of GeoMed, an Ashfield Company, part of UDG Healthcare plc, during the preparation of this article.

\section{Open access}

This work is licensed under the Attribution-NonCommercial-NoDerivatives 4.0 Unported License. To view a copy of this license, visit http://creativecommons.org/licenses/by-nc-nd/4.0/

\section{References}

Papers of special note have been highlighted as: $\bullet$ of interest; $\bullet \bullet$ of considerable interest

1. Sharma SV, Bell DW, Settleman J et al. Epidermal growth factor receptor mutations in lung cancer. Nat. Rev. Cancer 7(3), 169-181 (2007).

2. Spicer JF, Rudman SM. EGFR inhibitors in non-small cell lung cancer (NSCLC): the emerging role of the dual irreversible EGFR/HER2 inhibitor BIBW 2992. Target Oncol. 5(4), 245-255 (2010).

3. Zhou C, Wu YL, Chen G et al. Erlotinib versus chemotherapy as first-line treatment for patients with advanced EGFR mutation-positive non-small-cell lung cancer (OPTIMAL, CTONG-0802): a multicentre, open-label, randomised, Phase III study. Lancet Oncol. 12(8), 735-742 (2011).

4. Zhou C, Wu YL, Chen G et al. Final overall survival results from a randomised, Phase III study of erlotinib versus chemotherapy as first-line treatment of EGFR mutation-positive advanced non-small-cell lung cancer (OPTIMAL, CTONG-0802). Ann. Oncol. 26(9), 1877-1883 (2015).

5. Rosell R, Carcereny E, Gervais R et al. Erlotinib versus standard chemotherapy as first-line treatment for European patients with advanced EGFR mutation-positive non-small-cell lung cancer (EURTAC): a multicentre, open-label, randomised Phase 3 trial. Lancet Oncol. 13(3), 239-246 (2012).

6. Wu YL, Zhou C, Liam CK et al. First-line erlotinib versus gemcitabine/cisplatin in patients with advanced EGFR mutation-positive non-small-cell lung cancer: analyses from the Phase III, randomized, open-label, ENSURE study. Ann. Oncol. 26(9), 1883-1889 (2015).

7. Mok TS, Wu YL, Thongprasert S et al. Gefitinib or carboplatin-paclitaxel in pulmonary adenocarcinoma. N. Engl. J. Med. 361(10), 947-957 (2009).

8. Mitsudomi T, Morita S, Yatabe Y et al. Gefitinib versus cisplatin plus docetaxel in patients with non-small-cell lung cancer harbouring mutations of the epidermal growth factor receptor (WJTOG3405): an open label, randomised Phase III trial. Lancet Oncol. 11(2), 121-128 (2010).

9. Maemondo M, Inoue A, Kobayashi K et al. Gefitinib or chemotherapy for non-small-cell lung cancer with mutated EGFR. N. Engl. J. Med. 362(25), 2380-2388 (2010).

10. Fukuoka M, Wu YL, Thongprasert $S$ et al. Biomarker analyses and final overall survival results from a Phase III, randomized, open-label, first-line study of gefitinib versus carboplatin/paclitaxel in clinically selected patients with advanced non-small-cell lung cancer in Asia (IPASS). J. Clin. Oncol. 29(21), 2866-2874 (2011).

11. Inoue A, Kobayashi K, Maemondo M et al. Updated overall survival results from a randomized Phase III trial comparing gefitinib with carboplatin-paclitaxel for chemo-naive non-small cell lung cancer with sensitive EGFR gene mutations (NEJ002). Ann. Oncol. 24(1), 54-59 (2013).

12. Seto T, Kato T, Nishio $\mathrm{M}$ et al. Erlotinib alone or with bevacizumab as first-line therapy in patients with advanced non-squamous non-small-cell lung cancer harbouring EGFR mutations (JO25567): an open-label, randomised, multicentre, Phase II study. Lancet Oncol. 15(11), 1236-1244 (2014).

13. Solca F, Dahl G, Zoephel A et al. Target binding properties and cellular activity of afatinib (BIBW 2992), an irreversible ErbB family blocker. J. Pharmacol. Exp. Ther. 343(2), 342-350 (2012).

14. Modjtahedi H, Cho BC, Michel MC et al. A comprehensive review of the preclinical efficacy profile of the ErbB family blocker afatinib in cancer. Naunyn Schmiedebergs Arch. Pharmacol. 387(6), 505-521 (2014).

15. Li D, Ambrogio L, Shimamura T et al. BIBW2992, an irreversible EGFR/HER2 inhibitor highly effective in preclinical lung cancer models. Oncogene 27(34), 4702-4711 (2008). 
16. Ninomiya $\mathrm{T}$, Takigawa N, Ichihara $\mathrm{E}$ et al. Afatinib prolongs survival compared with gefitinib in an epidermal growth factor receptor-driven lung cancer model. Mol. Cancer Ther. 12(5), 589-597 (2013).

17. Sequist LV, Yang JC, Yamamoto $\mathrm{N}$ et al. Phase III study of afatinib or cisplatin plus pemetrexed in patients with metastatic lung adenocarcinoma with EGFR mutations. J. Clin. Oncol. 31(27), 3327-3334 (2013).

18. Wu YL, Zhou C, Hu CP et al. Afatinib versus cisplatin plus gemcitabine for first-line treatment of Asian patients with advanced non-small-cell lung cancer harbouring EGFR mutations (LUX-Lung 6): an open-label, randomised Phase III trial. Lancet Oncol. 15(2), 213-222 (2014).

19. Yang JC, Wu YL, Schuler M et al. Afatinib versus cisplatin-based chemotherapy for EGFR mutation-positive lung adenocarcinoma (LUX-Lung 3 and LUX-Lung 6): analysis of overall survival data from two randomised, Phase III trials. Lancet Oncol. 16(2), 141-151 (2015).

20. Soria JC, Ohe Y, Vansteenkiste J et al. Osimertinib in untreated EGFR-mutated advanced non-small-cell-lung-cancer. N. Engl. J. Med. 378(2), 113-125 (2018).

- Randomized Phase III trial demonstrating improved progression-free survival (PFS) with osimertinib versus gefitinib or erlotinib in patients with EGFR mutation-positive non-small-cell-lung-cancer (NSCLC).

21. Arcila ME, Oxnard GR, Nafa K et al. Rebiopsy of lung cancer patients with acquired resistance to EGFR inhibitors and enhanced detection of the T790M mutation using a locked nucleic acid-based assay. Clin. Cancer Res. 17(5), 1169-1180 (2011).

22. Sequist LV, Waltman BA, Dias-Santagata D et al. Genotypic and histological evolution of lung cancers acquiring resistance to EGFR inhibitors. Sci. Transl. Med. 3(75), 75ra26 (2011).

23. Yang JC, Ahn MJ, Kim DW et al. Osimertinib in pretreated T790M-positive advanced non-small-cell lung cancer: AURA study Phase II extension component. J. Clin. Oncol. 35(12), 1288-1296 (2017).

24. Hochmair M, Schwab S, Burghuber O et al. P2.03-025 prevalence of EGFR T790M mutation in NSCLC patients after afatinib failure, and subsequent response to osimertinib. J. Thorac. Oncol. 12(11), S2137 (2017).

25. Wu SG, Liu YN, Tsai MF et al. The mechanism of acquired resistance to irreversible EGFR tyrosine kinase inhibitor-afatinib in lung adenocarcinoma patients. Oncotarget 7(11), 12404-12413 (2016).

26. Yun $\mathrm{CH}$, Mengwasser KE, Toms AV et al. The T790M mutation in EGFR kinase causes drug resistance by increasing the affinity for ATP. Proc. Natl Acad. Sci. USA 105(6), 2070-2075 (2008).

27. Mok TS, Wu YL, Ahn MJ et al. Osimertinib or platinum-pemetrexed in EGFR T790M-positive lung cancer. N. Engl. J. Med. 376(7), 629-640 (2017).

- The Phase III AURA3 study demonstrating significant PFS benefit with osimertinib versus chemotherapy in patients with T790M-positive tumors following progression on erlotinib, gefitinib or afatinib.

28. Janne PA, Yang JC, Kim DW et al. AZD9291 in EGFR inhibitor-resistant non-small-cell lung cancer. N. Engl. J. Med. 372(18), 1689-1699 (2015).

29. Lin Y, Wang X, Jin H. EGFR-TKI resistance in NSCLC patients: mechanisms and strategies. Am. J. Cancer Res. 4(5), 411-435 (2014).

30. Klempner SJ, Bazhenova LA, Braiteh FS et al. Emergence of RET rearrangement co-existing with activated EGFR mutation in EGFR-mutated NSCLC patients who had progressed on first- or second-generation EGFR TKI. Lung Cancer 89(3), 357-359 (2015).

31. Nurwidya F, Takahashi F, Murakami A et al. Epithelial mesenchymal transition in drug resistance and metastasis of lung cancer. Cancer Res. Treat. 44(3), 151-156 (2012).

32. Campo M, Gerber D, Gainor JF et al. Acquired resistance to first-line afatinib and the challenges of prearranged progression biopsies. $J$. Thorac. Oncol. 11(11), 2022-2026 (2016).

33. Manca P, Russano M, Pantano F et al. Change from lung adenocarcinoma to small cell lung cancer as a mechanism of resistance to afatinib. Oncotarget 8(35), 59986-59990 (2017).

34. Nishimura J, Miyamoto Y, Fujimoto $\mathrm{N}$ et al. Adenocarcinoma of the lung acquiring resistance to afatinib by transformation to small cell carcinoma: a case report. Case Rep. Oncol. 10(2), 666-670 (2017).

35. Minari R, Bordi P, Tiseo M. Third-generation epidermal growth factor receptor-tyrosine kinase inhibitors in T790M-positive non-small cell lung cancer: review on emerged mechanisms of resistance. Transl. Lung Cancer Res. 5(6), 695-708 (2016).

36. Ramalingam SS, Yang JC, Lee CK et al. Osimertinib as first-line treatment of EGFR mutation-positive advanced non-small-cell lung cancer. J. Clin. Oncol. 36(9), 841-849 (2018).

- To date, the only study to assess resistance mechanisms to first-line osimertinib in patients with EGFR mutation-positive NSCLC, demonstrating heterogeneous resistance mechanisms.

37. Le X, Puri S, Negrao MV et al. Landscape of EGFR -dependent and -independent resistance mechanisms to osimertinib and continuation therapy post-progression in EGFR-mutant NSCLC. Clin. Cancer Res. doi:10.1158/1078-0432.CCR-18-1542 (2018) (Epub ahead of print).

38. Tan CS, Kumarakulasinghe NB, Huang YQ et al. Third generation EGFR TKIs: current data and future directions. Mol. Cancer 17(1), 29 (2018). 
39. Thress KS, Paweletz CP, Felip E et al. Acquired EGFR C797S mutation mediates resistance to AZD9291 in non-small cell lung cancer harboring EGFR T790M. Nat Med. 21(6), 560-562 (2015).

40. Oxnard GR, Hu Y, Mileham KF et al. Assessment of resistance mechanisms and clinical implications in patients with EGFR T790M-positive lung cancer and acquired resistance to osimertinib. JAMA Oncol. 4(11), 1527-1534 (2018).

41. Yang Z, Yang N, Ou Q et al. Investigating novel resistance mechanisms to third-generation EGFR tyrosine kinase inhibitor osimertinib in non-small cell lung cancer patients. Clin. Cancer Res. 24(13), 3097-3107 (2018).

42. Ercan D, Choi HG, Yun CH et al. EGFR mutations and resistance to irreversible pyrimidine-based EGFR inhibitors. Clin. Cancer Res. 21(17), 3913-3923 (2015).

43. Burrell RA, Swanton C. Re-evaluating clonal dominance in cancer evolution. Trends Cancer 2(5), 263-276 (2016).

44. Nowell PC. The clonal evolution of tumor cell populations. Science 194(4260), 23-28 (1976).

45. Aparicio S, Caldas C. The implications of clonal genome evolution for cancer medicine. N. Engl. J. Med. 368(9), 842-851 (2013).

46. Burrell RA, McGranahan N, Bartek J et al. The causes and consequences of genetic heterogeneity in cancer evolution. Nature 501(7467), 338-345 (2013).

47. Gerlinger M, Rowan AJ, Horswell S et al. Intratumor heterogeneity and branched evolution revealed by multiregion sequencing. $N$. Engl. J. Med. 366(10), 883-892 (2012).

48. de Bruin EC, McGranahan N, Mitter R et al. Spatial and temporal diversity in genomic instability processes defines lung cancer evolution. Science 346(6206), 251-256 (2014).

49. Blakely CM, Watkins TBK, Wu W et al. Evolution and clinical impact of co-occurring genetic alterations in advanced-stage EGFR-mutant lung cancers. Nat. Genet. 49(12), 1693-1704 (2017).

-• Comprehensive preclinical analysis of co-occuring genomic aberrations in EGFR mutation-positive NSCLC tumors.

50. Hata AN, Niederst MJ, Archibald HL et al. Tumor cells can follow distinct evolutionary paths to become resistant to epidermal growth factor receptor inhibition. Nat. Med. 22(3), 262-269 (2016).

51. Jamal-Hanjani M, Quezada SA, Larkin J et al. Translational implications of tumor heterogeneity. Clin. Cancer Res. 21(6), 1258-1266 (2015).

52. Rolfo C, Mack PC, Scagliotti GV et al. Liquid biopsy for advanced non-small cell lung cancer (NSCLC): a statement paper from the IASCL. J. Thorac. Oncol. 13(9), 1248-1268 (2018).

53. Torga G, Pienta KJ. Patient-paired sample congruence between 2 commercial liquid biopsy tests. JAMA Oncol. 4(6), 868-870 (2018).

54. Cancer Genome Atlas Research Network. Comprehensive genomic characterization of squamous cell lung cancers. Nature 489(7417), 519-525 (2012).

55. Su KY, Chen HY, Li KC et al. Pretreatment epidermal growth factor receptor (EGFR) T790M mutation predicts shorter EGFR tyrosine kinase inhibitor response duration in patients with non-small-cell lung cancer. J. Clin. Oncol. 30(4), 433-440 (2012).

56. Maheswaran S, Sequist LV, Nagrath S et al. Detection of mutations in EGFR in circulating lung-cancer cells. N. Engl. J. Med. 359(4), 366-377 (2008).

57. Ye X, Zhu ZZ, Zhong L et al. High T790M detection rate in TKI-naive NSCLC with EGFR sensitive mutation: truth or artifact? J. Thorac. Oncol. 8(9), 1118-1120 (2013).

58. Ramirez M, Rajaram S, Steininger RJ et al. Diverse drug-resistance mechanisms can emerge from drug-tolerant cancer persister cells. Nat. Commun. 7(1), 10690 (2016).

59. Sharma SV, Lee DY, Li B et al. A chromatin-mediated reversible drug-tolerant state in cancer cell subpopulations. Cell 141(1), 69-80 (2010).

60. Hangauer MJ, Viswanathan VS, Ryan MJ et al. Drug-tolerant persister cancer cells are vulnerable to GPX4 inhibition. Nature 551(7679), 247-250 (2017).

61. Terai H, Kitajima S, Potter DS et al. ER stress signaling promotes the survival of cancer "persister cells" tolerant to EGFR tyrosine kinase inhibitors. Cancer Res. 78(4), 1044-1057 (2018).

62. Bhang HE, Ruddy DA, Krishnamurthy Radhakrishna V et al. Studying clonal dynamics in response to cancer therapy using high-complexity barcoding. Nat. Med. 21(5), 440-448 (2015).

63. Lee CK, Wu YL, Ding PN et al. Impact of specific epidermal growth factor receptor (EGFR) mutations and clinical characteristics on outcomes after treatment with EGFR tyrosine kinase inhibitors versus chemotherapy in EGFR-mutant lung cancer: a meta-analysis. J. Clin. Oncol. 33(17), 1958-1965 (2015).

64. Kobayashi Y, Mitsudomi T. Not all epidermal growth factor receptor mutations in lung cancer are created equal: perspectives for individualized treatment strategy. Cancer Sci. 107(9), 1179-1186 (2016).

-• Comprehensive in vitro analysis of the sensitivity of EGFR mutations, including uncommon and compound mutations, to different EGFR tyrosine kinase inhibitors (TKIs). 
65. Tu HY, Ke EE, Yang JJ et al. A comprehensive review of uncommon EGFR mutations in patients with non-small cell lung cancer. Lung Cancer 114(1), 96-102 (2017).

66. Kohsaka S, Nagano M, Ueno T et al. A method of high-throughput functional evaluation of EGFR gene variants of unknown significance in cancer. Sci. Transl. Med. 9(416), pii:eaan6566 (2017).

-• Comprehensive in vitro analysis of the sensitivity of EGFR mutations, including uncommon and compound mutations, to different EGFR TKIs.

67. Kim EY, Cho EN, Park HS et al. Compound EGFR mutation is frequently detected with co-mutations of actionable genes and associated with poor clinical outcome in lung adenocarcinoma. Cancer Biol. Ther. 17(3), 237-245 (2016).

68. Wu JY, Yu CJ, Chang YC et al. Effectiveness of tyrosine kinase inhibitors on "uncommon" epidermal growth factor receptor mutations of unknown clinical significance in non-small cell lung cancer. Clin. Cancer Res. 17(11), 3812-3821 (2011).

69. Kobayashi S, Canepa HM, Bailey AS et al. Compound EGFR mutations and response to EGFR tyrosine kinase inhibitors. J Thorac. Oncol. 8(1), 45-51 (2013).

70. Khorashad JS, Kelley TW, Szankasi P et al. BCR-ABL1 compound mutations in tyrosine kinase inhibitor-resistant CML: frequency and clonal relationships. Blood 121(3), 489-498 (2013).

71. Park K, Tan EH, O’Byrne K et al. Afatinib versus gefitinib as first-line treatment of patients with EGFR mutation-positive non-small-cell lung cancer (LUX-Lung 7): a Phase 2B, open-label, randomised controlled trial. Lancet Oncol. 17(5), 577-589 (2016).

- Randomized Phase IIb trial demonstrating improved PFS and time to treatment failure with afatinib versus gefitinib in patients with EGFR mutation-positive NSCLC.

72. Paz-Ares L, Tan EH, O'Byrne $\mathrm{K}$ et al. Afatinib versus gefitinib in patients with EGFR mutation-positive advanced non-small-cell lung cancer: overall survival data from the phase IIb LUX-Lung 7 trial. Ann. Oncol. 28(2), 270-277 (2017).

- Final overall survival analysis of LUX-Lung 7. Overall survival was very encouraging in patients who received sequential afatinib and osimertinib.

73. Sequist L, Wu Y, Schuler M et al. 1349P - subsequent therapies post-afatinib among patients (pts) with EGFR mutation-positive (EGFRm+) NSCLC in LUX-Lung (LL) 3, 6 and 7. Ann Oncol. 28(Suppl. 5), v460-v496 (2017).

74. Wu YL, Cheng Y, Zhou X et al. Dacomitinib versus gefitinib as first-line treatment for patients with EGFR-mutation-positive non-small-cell lung cancer (ARCHER 1050): a randomised, open-label, Phase III trial. Lancet Oncol. 18(11), 1454-1466 (2017).

- Randomized Phase III trial demonstrating improved PFS with dacomitinib versus gefitinib in patients with EGFR mutation-positive NSCLC.

75. Mok TS, Cheng Y, Zhou X et al. Improvement in overall survival in a randomized study that compared dacomitinib with gefitinib in patients with advanced non-small-cell lung cancer and EGFR-activating mutations. J. Clin. Oncol. 36(22), 2244-2250 (2018).

76. Pilotto $\mathrm{S}$, Rossi A, Vavala $\mathrm{T}$ et al. Outcomes of first-generation EGFR-TKIs against non-small-cell lung cancer harboring uncommon EGFR mutations: a post hoc analysis of the BE-POSITIVE study. Clin. Lung Cancer 19(1), 93-104 (2018).

77. Klughammer B, Brugger W, Cappuzzo F et al. Examining treatment outcomes with erlotinib in patients with advanced non-small cell lung cancer whose tumors harbor uncommon EGFR mutations. J. Thorac. Oncol. 11(4), 545-555 (2016).

78. Watanabe S, Minegishi $\mathrm{Y}$, Yoshizawa $\mathrm{H}$ et al. Effectiveness of gefitinib against non-small-cell lung cancer with the uncommon EGFR mutations G719X and L861Q. J. Thorac. Oncol. 9(2), 189-194 (2014).

79. Yang JC, Sequist LV, Geater SL et al. Clinical activity of afatinib in patients with advanced non-small-cell lung cancer harbouring uncommon EGFR mutations: a combined post-hoc analysis of LUX-Lung 2, LUX-Lung 3, and LUX-Lung 6. Lancet Oncol. 16(7), 830-838 (2015).

80. Wu Y, Tu H, Feng J et al. P3.01-036 a Phase IIIb open-label, single-arm study of afatinib in EGFR TKI-naive patients with EGFRm+ NSCLC: an interim analysis. J. Thorac. Oncol. 12(11), S2214 (2017).

81. Kim Y, Sun J, Park K et al. P3.01-023 first-line afatinib for non-small cell lung cancer in real world practice. J. Thorac. Oncol. 12(11), S2209 (2017).

82. Saxon JA, Sholl LM, Janne PA. EGFR L858M/L861Q cis mutations confer selective sensitivity to afatinib. J. Thorac. Oncol. 12(5), 884-889 (2017).

83. Hochmair MJ, Morabito A, Hao D et al. Sequential treatment with afatinib and osimertinib in patients with EGFR mutation-positive non-small-cell lung cancer: an observational study. Future Oncol. 14(27), 2861-2874 (2018).

84. Tamiya M, Tamiya A, Suzuki $\mathrm{H}$ et al. Which is better EGFR-TKI followed by osimertinib between afatinib and gefitinib/erlotinib? Ann. Oncol. 29(Suppl. 8), viii493-viii547 (2018).

85. Huang WL, Chen YL, Yang SC. Liquid biopsy genotyping in lung cancer: ready for clinical utility? Oncotarget 8(11), 1859018608 (2017).

86. Hirsh V. Turning EGFR mutation-positive non-small-cell lung cancer into a chronic disease: optimal sequential therapy with EGFR tyrosine kinase inhibitors. Ther. Adv. Med. Oncol. 10(1), 1758834017753338 (2018). 